\title{
EL RECURSO TURÍSTICO NATURAL Y CULTURAL DEL DISTRITO DE MARAS COMO ALTERNATIVA DE DESARROLLO TURÍSTICO
} the touris resource of the maras district as an alternative of tourist de velopment

\author{
Aydeé Flores Contreras ${ }^{1}$ \\ ${ }^{1}$ Universidad Andina del Cusco, Cusco, Perú. \\ afloresc@uandina.edu.pe
}

\begin{abstract}
Resumen
Perú, país megadiverso de gran potencial natural y cultural. El distrito de Maras cuenta con recursos turísticos poco difundidos; sin embargo, de gran interés. El objetivo del presente trabajo de investigación fue identificar el potencial turístico natural y cultural del distrito de Maras como alternativa de desarrollo turístico del distrito, para lo cual se realizó una inventariación, categorización y jerarquización del recurso turístico de la zona. De igual forma se determinó la demanda turística del distrito a partir de entrevistas a turistas y pobladores. Fruto de este análisis, se pudo determinar que el distrito de Maras cuenta con atractivos turísticos de nivel de jerarquía II, lo que le permitirá ser ofertado en diversos paquetes turísticos. La presente investigación concluye que sí es factible diversificar el potencial turístico de este distrito de como alternativa de desarrollo turístico.

Palabras clave: Potencial turístico, recursos turísticos, metodología de evaluación de recursos turísticos
\end{abstract}

\begin{abstract}
Peru is a mega diverse country with great natural and cultural potential. The district of Maras has little-known tourist resources, however of great interest. The objective of this research was to identify the natural and cultural tourism potential of the Maras district as an alternative to tourism development in this district, for which an inventory, categorization and ranking of the tourist resource of the area was carried out. Likewise, the tourist demand of the district was determined from interviews with tourists and residents. As a result of this analysis, it was determined that the district of Maras has tourist attractions of hierarchy level II, which will allow it to be offered in different packages tourist. The present investigation concludes that it is feasible that it is a tourist resource to diversify the tourism potential of the district of Maras as an alternative for tourism development.
\end{abstract}

Keywords: Tourism potential, tourism resources, tourism resources evaluation methodology,

Citar como: Flores, A. (2018). El recurso turístico natural y cultural del distrito de maras como alternativa de desarrollo turístico. Rev Yachay, $7(1), 310-315$.

Recibido: 16-09-2018; Aceptado 07-12-2018

\section{Introducción}

El turismo se desarrolla en base a los atractivos turísticos con los que cuenta un país, los que deben ser debidamente aprovechados en base a una identificación cultural por parte de las instituciones que tienen que velar por la protección, conservación y uso racional de sus recursos. Por consiguiente, el turismo es fascinante y engrandece económica, social y culturalmente al país que hace uso racional de los recursos turísticos, lo que permite conocer, conservar, valorar y difundir el patrimonio turístico con el que cuenta, en este caso el distrito de Maras.

Cusco es considerado como uno de los destinos turísticos por excelencia, a nivel nacional e internacional, privilegiado debido a la potencialidad de los recursos turísticos con los que cuenta, heredera de una cultura milenaria de sabiduría, tecnología y costumbres.

Perú, país de gran potencial, posee recursos turísticos que son las expresiones de la naturaleza, la riqueza arqueológica, expresiones históricas materiales e inmateriales de gran tradición y valor que constituyen la base del producto turístico. El desarrollo de la actividad turística en el distrito de Maras por sus recursos potenciales, viene a constituir la base fundamental del desarrollo de esta actividad, siendo imprescindible su análisis y evaluación; para determinar dicha potencialidad y, en consecuencia, establecer su viabilidad como soporte para el desarrollo turístico, aunque todavía no han sido considerados de manera sustentable en la planificación del desarrollo turístico. La problemática principal es que estos recursos turísticos son escasamente difundidos para los visitantes y poco conocidos por los mismos pobladores. Para ordenar estos recursos en el espacio turístico del distrito de Maras será necesario dar a conocer el potencial de tales

Rev Yachay volumen (7) Numero (1), enero-diciembre 2018 
recursos y de acuerdo con la nueva tendencia turística en general, debe revelarse un cambio de enfoque para enfrentar su diseño. El más importante de ellos, es la temática de los inventarios de los recursos turísticos, que fue abordada en 1978 por la Organización Mundial de Turismo (OMT), ante las complejidades reflejadas en los diferentes estudios realizados al respecto en la década de los setenta, sobre todo, en México, España y otros países de Europa y América (Organización Mundial de Turismo, 1978).

Para determinar el inventario del patrimonio turístico del Distrito de Maras se utilizó el modelo OEA-CICATUR; mientras que, para el análisis de la oferta turística se necesitó una búsqueda de documentación y una investigación de campo, proceso que se realizó mediante la identificación y recolección de información de cada lugar considerado como atractivo turístico actual o potencial. Asimismo, el Mincetur, aportó diversas experiencias útiles en la valoración de estos recursos para su puesta en valor o rediseño, aplicándose un proceso metodológico de inventariación, categorización, jerarquización y evaluación analítica e integrada que permite establecer de manera objetiva su grado de atracción y sustentabilidad, e inclusive se logra dar la jerarquía adecuada para su posterior puesta en valor. Es más, se observó la evolución del turismo en este distrito, se constató que, actualmente, no se cuentan con instrumentos y herramientas que permitan el ordenamiento de sus recursos turísticos, que den a conocer sus reales potenciales actuales y futuros, tendientes a lograr el desarrollo socio económico de sus pobladores (MINCETUR, 7)

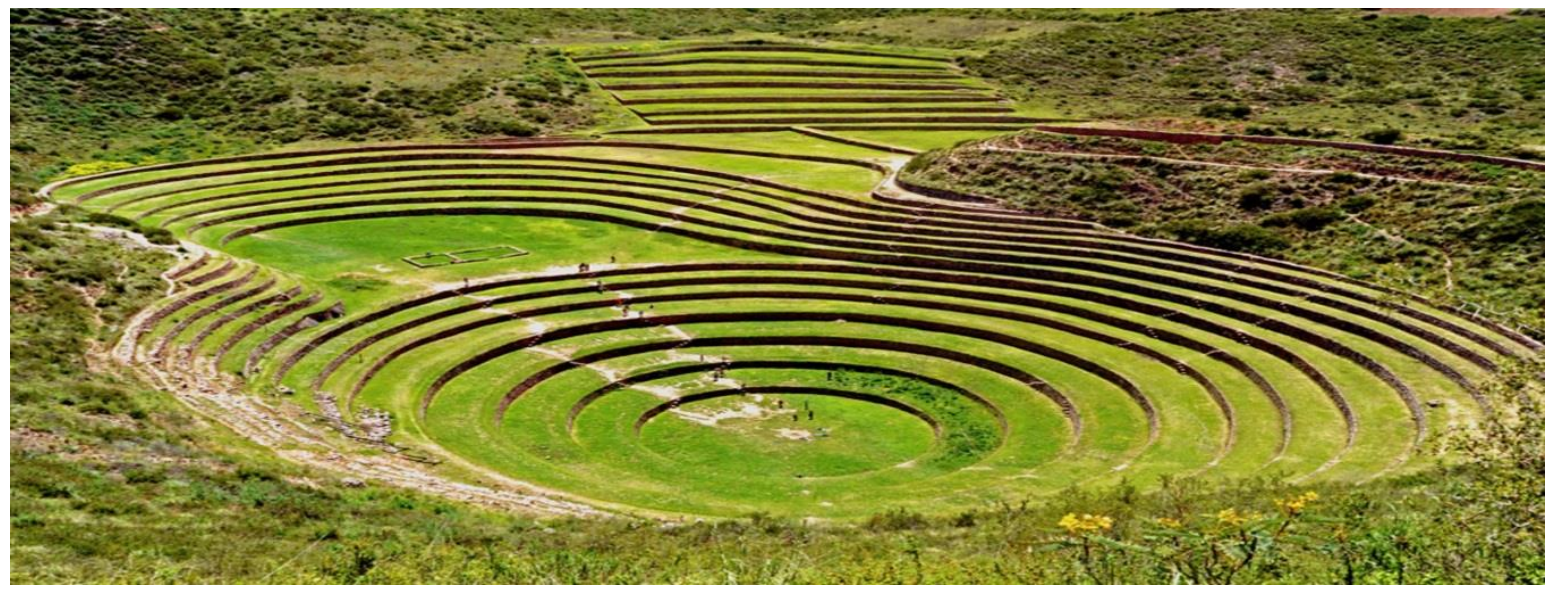

\section{Materiales y métodos}

\section{Delimitación turística del área de estudio}

El área de estudio del Distrito de Maras está enmarcada en las ciudades de Urubamba, Chinchero, vía Izcuchaca y Huarocondo, a una altitud de 3380 msnm a $48 \mathrm{Km}$ de la ciudad del Cusco.

\section{Antecedentes históricos del distrito de Maras}

Fundada en el año de 1556 por Pedro Ortiz de Orué, con el nombre de Villa San Francisco de Maras, lugar donde se establecieron los nobles del Imperio Incaico desplazados del Cusco. Destacan en el poblado más de 300 viviendas con sus hermosas portadas de los siglos XVI al XX. Destacan también sus iglesias, que conservan pinturas de los siglos XVII, XVIII y XIX, de artistas renombrados como Antonio Sinchi Roca.

En términos urbanísticos, arquitectónicos y funcionales, se dijo que Maras mantiene la estructura de las construcciones españolas donde predomina su plaza rodeada de construcciones públicas y privadas que gobiernan al poblado. Maras es mucho más que un espacio urbano; es desde su origen, una ciudad heterogénea que requiere salir del anonimato y del olvido de algunas autoridades, quienes no consideran la importancia de poseer recursos turísticos potenciales que, por desconocimiento, están perdiendo la oportunidad de desarrollo turístico que mejore su condición de vida.

De esta manera, pueden argumentarse tres factores potenciales que influyen actualmente en el desarrollo de la actividad turística de este distrito:

1. La presencia de un entorno paisajístico natural envidiable, rodeado por las cadenas montañosas del Vilcanota, donde destacan los nevados del Chicón, la Verónica, el Puma Huanca y el extraordinario Valle Sagrado de los Incas.

Rev Yachay volumen (7) Numero (1), enero-diciembre 2018 
2. El patrimonio turístico arquitectónico y urbanístico, invalorables debido a la presencia del grupo arqueológico de Moray, Checocc, Pulpituyuc y las Salineras de Maras.

3. La población, netamente mestiza, donde destacan las casas con los escudos mobiliarios de los siglos XVII, XVIII y XIX, y actuales.

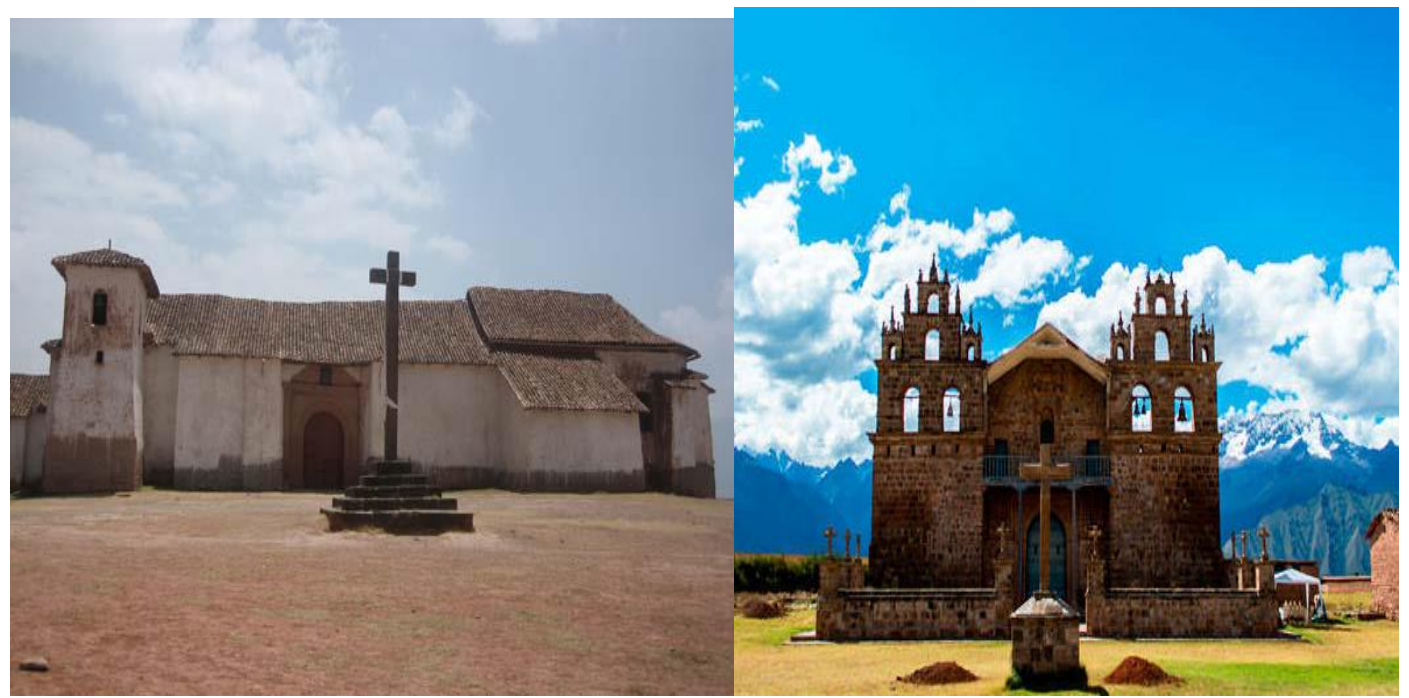

\section{Métodos y técnicas del Inventario de los recursos turísticos del distrito de Maras}

El conocimiento de la clasificación e inventario de los recursos existentes, a pesar de ser una condición necesaria, no es suficiente para conocer sus potenciales y el valor del potencial turístico de un área y no se mide únicamente por el número de atractivos que reúne, sino por la calidad de éstos. Por tanto, una vez que los recursos son clasificados, identificados e inventariados en categorías, tipos y subtipos.

Por las consideraciones expuestas, corresponde plantear la propuesta metodológica adecuada para inventariar, categorizar y jerarquizar el potencial del recurso turístico del distrito de Maras a través de las fichas de inventariación, evaluación y jerarquización que es una investigación descriptiva que estudió el recurso turístico in-situ dentro de su contexto real, clasificando cada uno de estos recursos según sus categorías considerando lo siguiente:

1. Se definió las categorías, tipos, subtipos y elementos de información a incluir para cada recurso turístico, para lo que se tuvo en cuenta los documentos modelos y manuales referidos a la elaboración de inventarios de recursos turísticos.

2. Se formuló un conjunto de tablas de inventariación, categorización y jerarquización acuerdo con la realidad del recurso turístico con la que cuenta el distrito de Maras.

3. Se recopiló la información secundaria, donde se consideraron todos los datos analizados, bibliografía existente, periódicos, revistas e información de organismos técnicos especializados.

4. Se llevó a cabo trabajo de campo, el que consistió en la identificación y/o verificación in situ de los recursos turísticos, tipos de medios de acceso y facilidades turísticas, para su reconocimiento, permitiendo la ampliación y/o ajuste de la información obtenida en la etapa anterior.

5. Del mismo modo, se llevaron a cabo entrevistas necesarias con representantes de la municipalidad de Maras y algunos miembros de las comunidades del distrito. Lo cual se complementa. según sea el caso, con la información que proporcionen los organismos e instituciones responsables de su cuidado.

6. Se procesó la información recopilada. En esta etapa se realizó la clasificación y ordenamiento de los recursos, de acuerdo con la categoría, previamente establecida acerca del tipo y/o subtipo que le corresponde. A cada uno se asignó la jerarquía.

Rev Yachay volumen (7) Numero (1), enero-diciembre 2018 


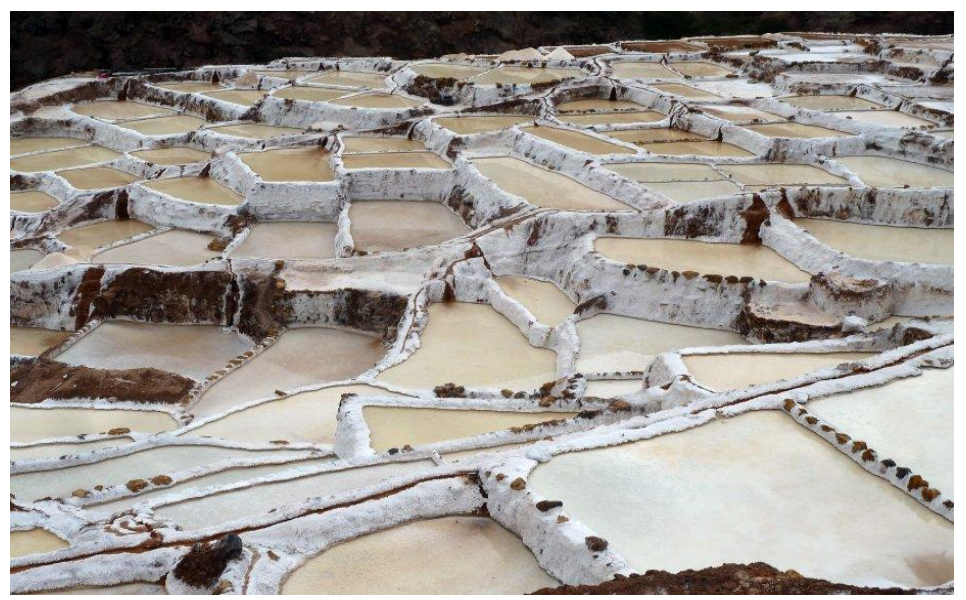

Resumen de resultados de la jerarquización del recurso turístico del distrito de Maras

\begin{tabular}{|c|c|c|}
\hline $\mathrm{N}^{\mathrm{o}}$ ficha & Nombre del atractivo & Puntos \\
\hline 01 & Nevado del Chicón & 3.00 \\
\hline 02 & Nevado de la Verónica & 3.00 \\
\hline 03 & Montaña de Aywayro & 1.00 \\
\hline 04 & Montaña de Checcoc & 1.00 \\
\hline 05 & Montana de Wañuymarka & 1.00 \\
\hline 06 & Pampa de Chillcapampa & 2.00 \\
\hline 07 & Pampas de Piscopata & 2.00 \\
\hline 08 & Pampas de Mahuaypampa & 2.00 \\
\hline 09 & Pampas de Anccoto & 2.00 \\
\hline 10 & Pampas de Ahuaco & 2.00 \\
\hline 11 & Pampas de Kaqllarakay & 2.00 \\
\hline 12 & Riachuelo de Rauccas & 1.00 \\
\hline 13 & Riachuelo de Pfauchi & 1.00 \\
\hline 14 & Riachuelo de Pitic & 1.00 \\
\hline 15 & Quebrada de Huaruray & 3.00 \\
\hline 16 & Quebrada de Pulpituyuc & 3.00 \\
\hline 17 & Quebrada de Pitic & 1.00 \\
\hline 18 & Camino pintoresco Maras - Pulpituyuc - Moray & 3.00 \\
\hline 19 & Camino pintoresco Maras Salineras & 3.00 \\
\hline 20 & Iglesia de Tiobamba & 3.00 \\
\hline 21 & Plaza de Armas de Maras & 1.00 \\
\hline
\end{tabular}

Rev Yachay volumen (7) Numero (1), enero-diciembre 2018 


\begin{tabular}{|c|c|c|}
\hline 22 & Pueblo de Maras & 2.00 \\
\hline 23 & Iglesia de Maras & 3.00 \\
\hline 24 & Capilla Punku Mayor & 1.00 \\
\hline 25 & Capilla Jesús de Nazareno & 2.00 \\
\hline 26 & Portada de Pedro Ortiz de Orué & 2.00 \\
\hline 27 & Portada Sinchi roca & 2.00 \\
\hline 28 & Portada Sancho Uscapaucar & 2.00 \\
\hline 29 & Portada Jesuítica & 2.00 \\
\hline 30 & Portada San Francisco de Asís. & 2.00 \\
\hline 31 & Portada Sol y Luna & 2.00 \\
\hline 32 & Portada San José & 2.00 \\
\hline 33 & Portada de las Estrellas & 2.00 \\
\hline 34 & Portada Jesús María y José & 2.00 \\
\hline 35 & Portada de los Querubines & 2.00 \\
\hline 36 & Portada Ortiz de Orué “A” & 2.00 \\
\hline 37 & Portada San Miguel y San Rafael & 2.00 \\
\hline 38 & Grupo arqueológico Moray- sector Quechuyuc & 3.00 \\
\hline 39 & Moray Sector Intiwatana muyu & 2.00 \\
\hline 40 & Moray - Sector Herraje & 3.00 \\
\hline 41 & Moray _ Sector andenes solsticiales & 3.00 \\
\hline 42 & Moray__̃usta Hispana & 2.00 \\
\hline 43 & Grupo arqueológico de Checcoc & 3.00 \\
\hline 44 & Grupo arqueológico de Pultituyuc & 3.00 \\
\hline 45 & Salineras de Maras & 3.00 \\
\hline 46 & Pueblo de Pichincoto & 1.00 \\
\hline \multicolumn{2}{|c|}{ Total de Puntos } & 98.00 \\
\hline
\end{tabular}

$\mathrm{J}=\mathrm{E} \quad \mathrm{X} 1+\mathrm{X} 2+\mathrm{X} 3+\mathrm{X} 4+\mathrm{X} 5+\mathrm{X} 6+\mathrm{X} 7 \ldots+\mathrm{X} 46$, Reemplazando valores:

$\mathrm{J}=\mathrm{E} \quad \frac{98}{46}=2.130$. Jerarquía II

\section{Discusión de los Resultados}

Aunque existen criterios ambiguos y dispares, en la definición de los recursos turísticos, se logró arribar a conceptos sustentados en criterios válidos para este caso de estudio. 
Además, se demuestra que, de las diferentes clasificaciones de los recursos básicos analizados, se destaca la propuesta de la Organización de Estados Americanos (OEA) y del Mincetur. De los diferentes métodos y técnicas de evaluación analizados en el ámbito nacional e internacional orientados a evaluar los recursos turísticos, se concluyó que ninguno resultaba apropiado para el fin que propone esta investigación, por lo que se determinaron los elementos válidos aportados por los mismos para incorporarlos a los métodos y técnicas del recurso turístico en el distrito de Maras.

Con la finalidad de subsanar el tradicional déficit con la mayor parte de los métodos y técnicas analizados y comentados sobre los recursos básicos, donde el objetivo principal de ellos no es la valoración del potencial turístico de un recurso concreto, sino determinar el valor de diversas zonas con el fin de establecer un orden de prioridad en las labores de planificación y desarrollo del turismo.

\section{Conclusiones}

1. Se ha demostrado que los recursos turísticos con los que cuenta el distrito de Maras son potenciales y tienen la jerarquía de II puntos, lo que sí permite que su inserción dentro de los circuitos turísticos con los que cuenta el departamento del Cusco.

2. Se ha evidenciado del resultado de la investigación que, a través del proceso metodológico de inventariación, evaluación y jerquización del recurso turístico del distrito de Maras, se motivará al desplazamiento de nuevas corrientes turísticas, permitiendo a los pobladores incursionar en el desarrollo de la actividad turística, como una nueva alternativa de desarrollo socio económico.

\section{Referencias Bibliográficas}

Boullon, R. C. Planificación del espacio turístico. México D.F: Trillas, 1998

Leno Cerro, F. Técnicas de evaluación del potencial turístico. Madrid: Ministerio de Industria, Comercio y Turismo. Dirección General de Política turística, 1993.

Ley $\mathrm{N}^{\circ} 29408$ - Ley General de Turismo, Anexo Nº 2 Glosario, punto 7.

MINCETUR. (2008). Manual para la formulación del inventario de recursos turísticos a nivel nacional. Lima. San Isidro. Viceministerio de Turismo.

MINCETUR. (2006). Manual para la formulación del inventario de recursos turísticos a nivel nacional.

Ministerio de Economía y Finanzas SNIP PERU. Inversión Pública de Calidad: Programa de Capacitación: Guía Metodológica de Identificación, Formulación y Evaluación Social.

Ministerio de Industria, Comercio Interior, Turismo e Integración - MICTI (1992). Guía Metodológica para la Formulación y Evaluación del Patrimonio Turístico Nacional. Lima.

Organización Mundial de Turismo. Evaluación de los recursos turísticos. Madrid: Organización Mundial de Turismo, 1978.

Organización de Estados Americanos, OEA. (1978). Metodología de inventario turístico en Quito. Curso Interamericano de Planificación del Desarrollo Turístico. México D. F.: Organización de Estados Americanos, 1978.

Organización de Estados Americanos OEA Cicatur. Centro Interamericano de Capacitación Turística. México (1996).

Rev Yachay volumen (7) Numero (1), enero-diciembre 2018 